\title{
Case series of thromboembolic complications in childhood nephrotic syndrome: Hacettepe experience
}

\author{
Betul Tavil · Fehime Kara $\cdot$ Rezan Topaloglu • \\ Selin Aytac · Sule Unal • Baris Kuskonmaz • \\ Mualla Cetin · Nesrin Besbas • Fatma Gumruk
}

Received: 18 March 2014/ Accepted: 16 June 2014/Published online: 4 July 2014

(C) Japanese Society of Nephrology 2014

\begin{abstract}
Background The aim of this study was to describe the incidence and contributory risk factors for thromboembolic complications in children with nephrotic syndrome (NS) and thrombosis.

Methods Among 188 children with the diagnosis of NS (80 girls; mean age: $12.6 \pm 5.4$ years) followed up in our hospital for the last 5 years, 17 (9.0\%) children (16 boys) identified as having thromboembolic complications. All 17 children with NS and thrombosis were screened for laboratory risk factors for thrombosis. The diagnosis was confirmed by cranial magnetic resonance imaging, doppler ultrasonography, and echocardiography.

Results Among 17 children with thrombosis, 14 (82.3\%) were found to have focal segmental glomerulosclerosis (FSGS) as underlying pathology by renal biopsy. The mean age of the thrombotic children was $4.5 \pm 3.2$ years at the diagnosis of NS and that was $7.1 \pm 4.9$ years at the time of thrombosis. The mean time from NS diagnosis to the first thrombosis development was $2.6 \pm 2.3$ years. Thrombosis occurred during the first year of NS in 9/17 (52.9\%) children. Most of the children (88.2\%) had venous thrombosis. Among the screened risk factors, high factor VIII level $(64.7 \%)$ was the leading factor followed by decreased antithrombin III level (29.4\%). Furthermore, 4
\end{abstract}

B. Tavil $\cdot$ S. Aytac $\cdot$ S. Unal · B. Kuskonmaz $\cdot$ M. Cetin F. Gumruk

Pediatric Hematology Unit, Hacettepe Univesity Faculty of Medicine, Sihhiye, 06100 Ankara, Turkey

F. Kara · R. Topaloglu $(\bowtie) \cdot$ N. Besbas

Pediatric Nephrology Unit, Hacettepe Univesity Faculty of

Medicine, Sihhiye, 06100 Ankara, Turkey

e-mail: rezantopaloglu@hacettepe.edu.tr children had central venous catheters and 2 had infection as clinical risk factors for thrombosis.

Conclusion In this case series, subtype of FSGS, active disease state of NS, central venous catheters, and some inherited and acquired thrombotic risk factors have been identified as contributory factors for the development of thrombosis in children with NS.

Keywords Thrombosis - Complication - Nephrotic syndrome $\cdot$ Children

\section{Introduction}

Nephrotic syndrome (NS) is characterized by a triad of proteinuria, edema, and hypoalbuminemia, which has an incidence of 2-7 per 100000 children. Childhood NS is most commonly caused by one of two idiopathic diseases: minimal-change nephrotic syndrome (MCNS) and focal segmental glomerulosclerosis (FSGS). MCNS, the most common form of idiopathic NS comprising $70-80 \%$ of all cases, is usually responsive to standard steroid treatment. Steroid-resistant NS presents mainly as FSGS, which represents about $20 \%$ of children with idiopathic NS. Common complications of NS and its treatment include infection, bone mineral loss, acute renal failure, and thromboembolism (TE). Although modern anticoagulant and thrombolytic therapies may decrease the risk of TErelated mortality, TE remains one of the most serious and life-threatening complication in adults and children with NS. The incidence of TE has been reported as approximately $20-30 \%$ in adults and $1.8-5 \%$ in children with NS [1-4]. Diagnosis and treatment of TE are important in the management of children with NS. The aim of this study was to describe the incidence and contributory risk factors 
for thromboembolic complications in children with NS and thrombosis.

\section{Materials and methods}

Among 188 children with the diagnosis of NS (80 girls and 108 boys; mean age: $12.6 \pm 5.4$ years) followed up at Hacettepe University Faculty of Medicine Pediatric Nephrology Department between July 2008 and March 2013, 17 children [(16 (94.1\%) boys and 1 (5.9\%) girl] were identified to have thromboembolic events. The medical records of these 17 children with thrombosis were reviewed retrospectively for inherited and laboratory risk factors for thrombosis [protein C (PC), protein S (PS), and antithrombin III (ATIII), homocystein, lipoprotein a, triglyceride and cholesterol levels, antiphospholipid antibodies (APA), factor $\mathrm{V}$ Leiden, prothrombin, methylenetetrahydrofolate reductase (MTHFR) 677 and 1298 mutations, plasminogen activator inhibitor (PAI) polymorphisms, fibrinogen, factors II, V, VII, VIII, IX, XI, and XII levels] at the time of thrombosis. Children with primary NS between the ages of 2 and 6 years were assumed to have MCNS and were given corticosteroid (CS) therapy without histopathological evaluation. The treatment regimen consisted of prednisone $(2 \mathrm{mg} / \mathrm{kg} / \mathrm{day} ; 2$ doses; maximum $60 \mathrm{mg} /$ day) given orally for 4 weeks, followed by alternate-day therapy for 4 weeks; the alternate dose was then tapered for 8-12 weeks and then, stopped. Response to CS therapy was defined as disappearance of proteinuria $\left(<4 \mathrm{mg} / \mathrm{m}^{2}\right.$ per day on 3 consecutive days), whereas children who had nephrotic range proteinuria and hypoalbuminemia at the end of 8 weeks of CS therapy were considered as steroid resistant. In all steroid-resistant children, percutaneous kidney biopsy was performed with ultrasonography guidance.

Medical charts and computer-based data were evaluated to obtain detailed information about clinical risk factors, radiological imaging methods, anticoagulation treatment, and long-term outcome of the children with NS and thrombosis. Children with clinical symptoms related to thrombosis were screened for thrombosis by imaging methods. Children with tachycardia, cyanosis and children having congenital heart diseases were screened by echocardiography. Cranial magnetic resonance imaging (MRI) was used for children having headache and/or vomiting and children with pulsatile fontanels during infancy period. Doppler ultrasonography (USG) was used for children with catheter dysfunction or abdominal discomfort or pain, edema and increase in temperature in extremities. Laboratory risk factors for thrombosis including plasma PC activity, free PS antigen, ATIII activity, APAs, homocystein, lipoprotein a, and factor levels were tested using the methods described previously [5]. Age-dependent charts were used for normal values of these parameters [6]. DNA was extracted from peripheral blood by standard procedures. FV Leiden (G1691A), prothrombin G20210A, MTHFR C677T, MTHFR A1298C mutations, and PAI polymorphisms were detected using the methods described earlier [7-10].

This study has been approved by ethical committee of our hospital (number: GO. 13/508). SPSS for Windows version 15.0 (SPSS Inc, Chicago, Illionis) was used for evaluation of the data. Student's $t$ test and 3-way Chisquared test were used for comparison of the data between thrombotic and non-thrombotic groups.

\section{Results}

Thromboembolic complications occurred in $17(9.0 \%)$ of 188 children with NS. The mean age of the 17 children was $4.5 \pm 3.2$ years ( 1 month-11 years; median 4.9 years) at the time of NS diagnosis and that was $7.1 \pm 4.9$ years (3 months-14 years; median 9 years) at the time of thrombosis. The mean time from NS diagnosis to the first thrombosis development was $2.6 \pm 2.3$ years (at day one11.5 years; median 2 years). Initial presentation of NS and thrombosis occurred at the same time in $2(11.7 \%)$ children. Thrombosis occurred during the first year of NS in 9 $(52.9 \%)$ children and after the first year of diagnosis in the other $8(47.1 \%)$ children. The mean follow-up duration was $7.0 \pm 3.9$ years ( 4 months-12.5 years) for these children. Age, gender, and type of NS were compared between thrombotic $(n: 17)$ and non-thrombotic $(n: 171)$ groups in Table 1. All children with thrombosis had active disease at the time of diagnosis. Among 17 children with thrombosis, $14(82.3 \%)$ were diagnosed as FSGS, 2 (11.8\%) were congenital NS, and only 1 (5.9\%) was MCNS. On the other hand, FSGS comprises 54 (31.5\%), MCNS comprises $96(56.1 \%)$, and congenital NS comprises $2(1.2 \%)$ children in non-thrombotic group $(p \leq 0.05)$. When thrombotic ( $n: 17)$ and non-thrombotic groups ( $n: 171)$ were compared, MCNS is a more common type of NS in nonthrombotic group and FSGS is a more common type of NS in thrombotic group (Table 1). FSGS and male gender were significantly higher in thrombotic group than in nonthrombotic group ( $p=0.004$ and $p<0.001$ ), respectively.

Thrombus localization

Among 17 children with thrombosis, 4 (23.5\%) had catheter-related thrombosis in jugular veins, $3(17.6 \%)$ had sagittal sinus thrombosis, $2(11.7 \%)$ had cerebral infarct, $2(11.7 \%)$ had portal venous thrombosis, 2 $(11.7 \%)$ had intracardiac (right ventricle and right atrium, 
Table 1 Comparison of children with and without thrombosis

\begin{tabular}{llll}
\hline $\begin{array}{l}\text { Total number of } \\
\text { children with NS } \\
(n: 188)\end{array}$ & $\begin{array}{l}\text { Patients with } \\
\text { thrombosis } \\
(n: 17)\end{array}$ & $\begin{array}{l}\text { Patients without } \\
\text { thrombosis } \\
(n: 171)\end{array}$ & $p$ \\
\hline Age (year) & $11.6 \pm 6.7$ & $12.5 \pm 5.4$ & $>0.05$ \\
Gender (girls/boys) & $1 / 16$ & $79 / 92$ & $<0.001$ \\
Types of NS & & & \\
MCNS & $1(5.9 \%)$ & $22+74^{\mathrm{a}}(56.1 \%)$ & $<0.05$ \\
FSGS & $14(82.3 \%)$ & $54(31.5 \%)$ & $<0.05$ \\
Congenital NS & $2(11.8 \%)$ & $2(1.2 \%)$ & $<0.05$ \\
DMS & - & $3(1.8 \%)$ & \\
MesPGN & - & $12(7.0 \%)$ & \\
MPGN & - & $1(0.6 \%)$ & \\
MGN & - & $1(0.6 \%)$ & \\
C1q nephropathy & - & $2(1.2 \%)$ & \\
\hline
\end{tabular}

MCNS Minimal-change nephrotic syndrome, FSGS Focal segmental glomerulosclerosis, DMS Diffuse mesangial sclerosis, MesPGN Mesangioproliferative glomerulonephritis, $M P G N$ Membranoproliferative glomerulonephritis, $M G N$ Membranous glomerulonephritis

a 22 children had renal biopsy and 74 did not have biopsy

respectively) thrombosis, $1(5.8 \%)$ had cerebral infarct and intracardiac thrombosis at tricuspid valve and patent ductus arteriosus, 1 (5.8 \%) had left sigmoid and transverse sinuses thrombosis, 1 (5.8 \%) had superficial right femoral vein thrombosis, and 1 (5.8 \%) had cephalic vein thrombosis. Therefore, only $2(11.8 \%)$ of them had arterial thrombosis (cerebral infarct), others had venous thrombosis. Localization of thrombosis, clinical and laboratory risk factors for the development of thrombosis, type and duration of anticoagulation therapy, and outcome in children with NS and thrombosis is shown in Table 2.

\section{Clinical and laboratory risk factors for thrombosis}

All children with NS and thrombosis had active disease and hyperlipidemia. Initial presentation of NS and thrombosis occurred at the same time in $2(11.7 \%)$ children (Case numbers 11 and 15 in Table 2). Several risk factors for thrombosis were identified in all children. Hyperlipidemia developed secondary to active NS was not considered as a risk factor. High factor VIII level was detected in 11/17 $(64.7 \%)$ children. High factor VIII level, the most common risk factor was followed by MTHFR C677T heterozygous mutation in $6 / 16(37.5 \%)$, decreased antithrombin III level in 5/17 (29.4\%), high homocystein level in 4/17 $(23.5 \%)$, high lipoprotein a level in 4/17 (23.5\%), high factor V levels in 4/17 (23.5\%), decreased protein C level in 3/17 (17.6\%), MTHFR A1298C heterozygous mutation in $3 / 13(23.0 \%)$, decreased protein $\mathrm{S}$ level in $2 / 17$ $(11.7 \%)$, anticardiolipin antibodies in 2/17 (11.7\%), factor V Leiden heterozygous mutation in 2/16 (12.5\%), PAI
(4G/5G) polymorphism in 2/13 (15.4\%), antiphospholipid antibodies in 1/17 (5.8\%), MTHFR C677T homozygous mutation in 1/16 (6.2\%), MTHFR A1298C homozygous mutation in $1 / 13(7.6 \%)$, and PAI $(4 \mathrm{G} / 4 \mathrm{G})$ polymorphism in 1/13 (7.6\%) child. Fibrinogen level was within normal range in all children and that is not shown in Table 2.

MTHFR 677 and 1298 compound heterozygous mutations in case number 17, MTHFR 677 homozygous mutation in case number 1, and MTHFR 1298 homozygous mutation in case number 5 were considered as important risk factors for thrombosis, whereas MTHFR 677 and 1298 heterozygous mutations were not considered as important risk factors for thrombosis.

Thus, three children had 1 , five children had 2, two children had 3, three children had 4, and three children had 5 risk factors for thrombosis. Furthermore, 4 children had central venous catheters and 2 had infection as clinical risk factors for thrombosis (Tables 2, 3).

Anticoagulant treatment and outcome

Children with venous thrombosis were treated with only low-molecular weight heparin (LMWH) treatment, whereas children with cerebral infarct were treated with LMWH and acetyl-salicylic acid (aspirin). Most of the children $(n: 12,70.1 \%)$ received only LMWH treatment. Two with cerebral infarct received both LMWH and acetyl-salicylic acid treatment and one child with renal transplantation received conventional heparin treatment which was followed by LMWH. LMWH treatment was monitored using anti-activated factor X (anti-FXa) assay and the target therapeutic anti-FXa level was $0.5-1.0$ $\mathrm{U} / \mathrm{mL}$. Thrombectomy was performed for 2 children. One of them needed thrombectomy due to compartment syndrome (Case 6) and LMWH treatment was given following thrombectomy. The other had thrombectomy to rescue transplanted kidney (Case 16). Conventional heparin followed by LMWH treatment was given after thrombectomy along with the anti-rejection therapy for case 16. The transplanted kidney was rescued with these therapies [11].

The duration of anticoagulation therapy was 6 months for most of the children $(n: 13,76.4 \%)$. Only $1(5.8 \%)$ child received 12 months therapy and $3(17.6 \%)$ children received 3 months therapy. Twelve children $(70.5 \%)$ had complete recovery from thrombosis, whereas $3(17.6 \%)$ had partial recovery with sequel and $2(11.7 \%)$ had died due to other causes except thrombosis (Tables 2, 3).

\section{Discussion}

Nephrotic syndrome has been considered as a thrombophilic or hypercoagulable state. Several mechanisms that 


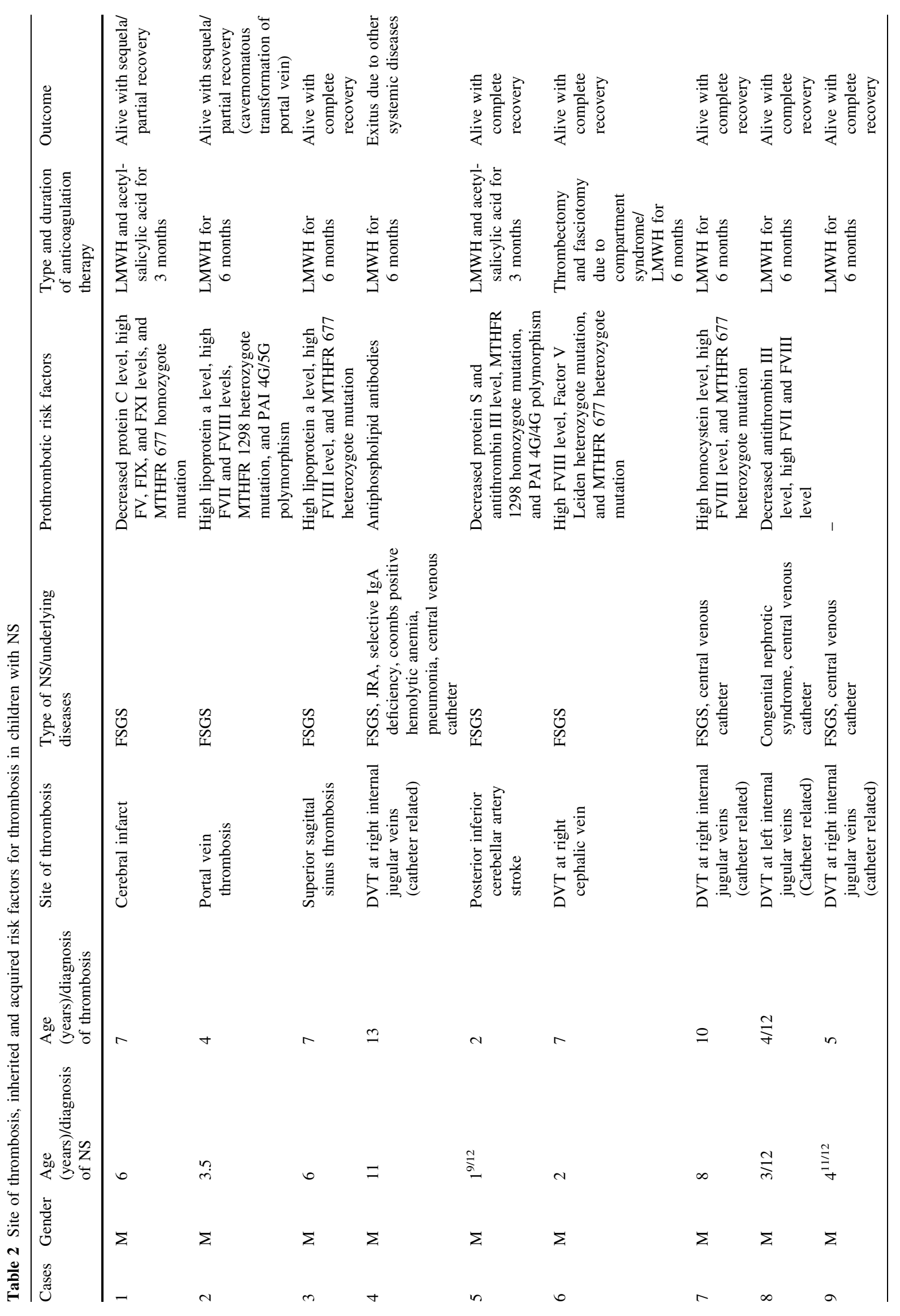




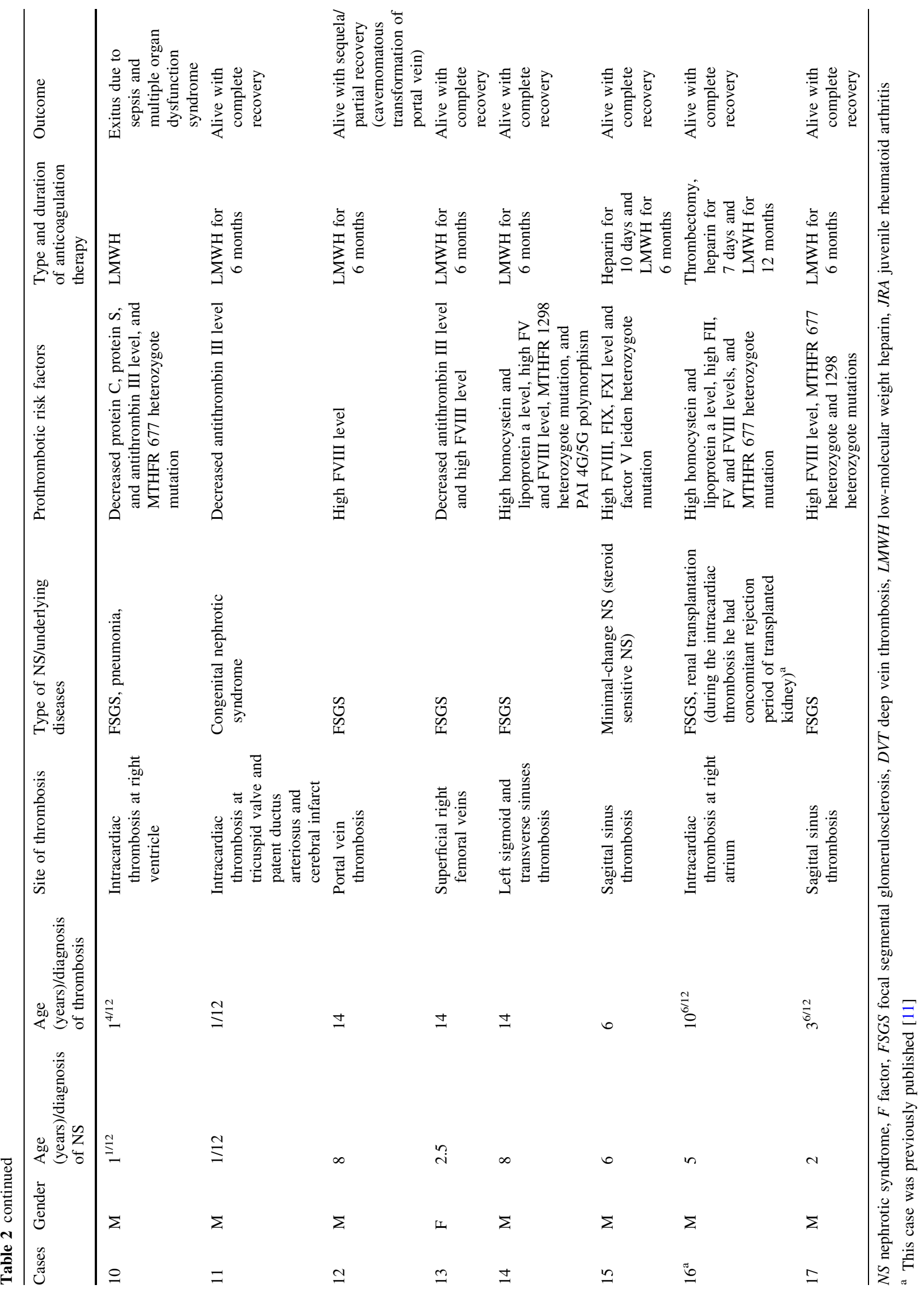


Table 3 Data of children with nephrotic syndrome and thrombosis

Number of children with thrombosis
Number of risk factors for each patient
Age (diagnosis of NS) (mean $\pm \mathrm{SD}$; range)
Age (diagnosis of thrombosis) (mean $\pm \mathrm{SD}$; range)
The duration of thrombosis development (mean $\pm \mathrm{SD}$; range)
Clinical and laboratory risk factors for thrombosis

\author{
$17 / 188$ \\ $2.4 \pm 1.2$ \\ $4.5 \pm 3.2$ years $(0.08-11.0)$ \\ $7.1 \pm 4.9$ years $(0.25-14.0)$ \\ $2.6 \pm 2.3$ years $(0-11.5)$ \\ Central venous catheters (4) \\ Infection (2) \\ Rejection episode of transplanted kidney (1) \\ Factor VIII $\uparrow(11 / 17)$ (median 275, range 53-170 \%) \\ ATIII $\downarrow$ (5/17) (median 44, range 80-120\%) \\ Lipoprotein a $\uparrow(4 / 17)$ (median 44 , range $0-30 \mathrm{mg} / \mathrm{dL}$ ) \\ Homocysteine $\uparrow$ (4/17) (median 23, range 5.5-17 micromol/L) \\ Factor V $\uparrow(4 / 17)$ (median 180, range 70-120 \%) \\ Protein C $\downarrow$ (3/17) (median 33, range 70-130 \%) \\ Protein $S \downarrow(2 / 17)$ (median 26, range 60-130\%) \\ Anticardiolipin antibodies (2/17) \\ Antiphospholipid antibodies (1/17) \\ FV G1691 A heterozygous mutation (2/16) \\ MTHFR C677T homozygous mutation (1/16) \\ MTHFR A1298C homozygous mutation (1/13) \\ MTHFR C677T/A1298C compound heterozygous mutation (1/16) \\ PAI (4G/5G) polymorphism (2/13) \\ PAI (4G/4G) polymorphism (1/13)
}

promote thrombosis have been identified in patients with NS. These mechanisms are divided into two categories. One of the risk factor is urinary loss of proteins that prevent thrombosis and the other is increased synthesis of factors that promote thrombosis. A loss of endogenous anticoagulant proteins: ATIII, protein $\mathrm{C}$ and protein $\mathrm{S}$ in the urine as a result of altered permselectivity of the glomerular basement membrane is one of the most important mechanisms in the development of thrombosis [12]. On the other hand, increased synthesis of fibrinogen, factors V and VIII, von Willebrand Factor (vWF), $\alpha 2$-plasmin inhibitor, and plasminogen activator inhibitor 1 (PAI-1) is another important factor that promotes thrombosis. It is thought that the increase in fibrinogen, factors V and VIII, vWF, and $\alpha 2$-macroglobulin is a reflection of increased acutephase synthesis. Hyperfibrinogenemia is a hepatic synthetic response to the hypoalbuminemia of NS, which promotes platelet aggregation, provides substrate for fibrin formation, and increases blood viscosity. Mild thrombocytosis and platelet hyper-reactivity also accompany NS. Platelet hyper-reactivity is multifactorial and can be attributed to increased levels of vWF, hyperfibrinogenemia, hypercholesterolemia, and hypoalbuminemia. At the level of fibrinolysis, NS is associated with a decrease in plasminogen levels. This decrement in plasminogen is accompanied by an increase in levels of PAI-1 and $\alpha 2$-plasmin inhibitor, all of which conspire to impair fibrin clearance and promote thrombus persistence. Additional factors including hereditary thrombotic risk factors, hospitalization, immobilization, infection, catheters, and diuretic therapy may also aggravate this pre-thrombotic state in patients with NS [13-15].

There are a lot of case reports about NS and thrombosis in childhood, whereas there are limited numbers of case series $[2,3,16,17]$. The overall incidence of thrombosis in children has been reported as $3 \%$. Kerlin et al. [3] reported that $9.2 \%$ of children have thromboembolic complications in a large cohort including children with primary and secondary NS. That high prevalence was attributed to the inclusion of children with secondary NS along with primary NS. In the present study, the prevalence of thromboembolic complications in primary NS was $9.0 \%$. This high incidence compared to previously reported incidence (1.8-5\%) [1-3] could be attributable that our center is a tertiary referral center admitting patients from all over the country and the rate of FSGS (31.6\%) is also high in our cohort. Among 17 children with NS and thrombosis, 14 $(82.3 \%)$ were FSGS, 2 (11.7\%) were congenital NS, and only $1(5.8 \%)$ was MCNS. All children with NS and thrombosis are steroid resistant except one with MCNS. 
Having FSGS seemed to increase thrombotic risk in children rather than membranous glomerulonephritis. Recently, two studies have reported that primary FSGS patients had high circulating endothelial cells and soluble biomarkers of endothelial dysfunction such as soluble thrombomodulin (sTM), vWF, soluble vascular cell adhesion molecule-1 (sVCAM-1), and sE-selectin [18, 19]. These markers are highest during the acute proteinuric phase of the disease and decrease as proteinuria reduces. Zhang et al. [19] showed that patients with thromboembolism (TE) have higher level of markers than patients without TE. These two studies have also showed that even during the inactive non-proteinuric phase, vWF and sVCAM-1 are persistently elevated, which may suggest a chronic activated state of endothelium and may promote the development of TE in FSGS [18, 19].

In our study, none of the children with NS and thrombosis were in remission at the diagnosis of thrombosis. Thrombosis occurred during the 1 year of NS diagnosis in $9(52.9 \%)$ children. In a study conducted by Kerlin BA [3], thrombosis occurred earlier than that seen in our cohort. The median time to the first thrombosis was 70.5 days after the diagnosis of NS in that study. In our study, the mean time from NS diagnosis to the first thrombosis development was $2.6 \pm 2.3$ years (range day one-11.5 years, median 2 years).

Two children presented as NS and thrombosis at the same time in our study (Case 11 and 15). One of them was the only case of MCNS who was positive for factor $\mathrm{V}$ Leiden heterozygote mutation. This inherited thrombophilic risk factor may be an important contributory factor for the development of thrombosis at the time of NS diagnosis (Tables 1,2).

Among 17 children with thrombosis, the most common localizations of thrombosis were catheter-related thrombosis in jugular veins $(n: 4,23.5 \%)$ and sino-venous thrombosis in dural veins $(n: 4,23.5 \%)$. Only $2(11.7 \%)$ of them had arterial thrombosis (cerebral infarct) and rest of them had venous thrombosis (Table 2). In other series, venous thrombosis is also more common than arterial thrombosis in children with NS [3, 15-17]. Catheter use is a well-known risk factor for thromboembolic complications in children. Kerlin BA [3] reported that $44.8 \%$ of thromboembolic complications were associated with catheters. Thus, central venous catheters should be removed as soon as possible in children with NS.

In the present study, the most common laboratory risk factors for thrombosis were high factor VIII level $(n: 11$, $64.7 \%)$ and decreased ATIII level $(n: 5,29.4 \%)$. On the other hand, inherited thrombophilia including factor $\mathrm{V}$ Leiden (FVL) heterozygote mutation $(n: 2,11.7 \%)$, MTHFR 677 homozygote mutation ( $n: 1,5.8 \%)$, MTHFR 1298 homozygote mutation (n:1, $5.8 \%)$, MTHFR 677 and
1298 compound heterozygote mutation $(n: 1,5.8 \%)$, PAI $(4 \mathrm{G} / 5 \mathrm{G})(n: 2,11.7 \%)$, and PAI $(4 \mathrm{G} / 4 \mathrm{G})$ polymorphisms $(n: 1,5.8 \%)$ were contributory risk factors for thrombosis (Table 3). The risk of developing thrombotic episodes for persons heterozygous for the FVL mutation is about 510 -fold and for those homozygous is 80 - 100-fold higher than the general population. The frequency of FVL mutation is $7.4 \%$ in healthy Turkish population [20]. The prothrombin G20210A mutation is the second most common inherited risk factor for thrombophilia. The frequency of prothrombin G20210A mutation is $2.3 \%$ in healthy Turkish population [20]. None of our patient carried prothrombin G20210A mutation, whereas FVL mutation was found in $11.8 \%$ of thrombotic group. The frequency of FVL mutation in the thrombotic group was slightly higher than that found in the healthy Turkish population (11.8 vs. $7.4 \%$ ). The frequency of MTHFR C677T heterozygous mutation was $28.0 \%$ and the frequency of homozygous mutation was $6.3 \%$ in healthy Turkish population. Then, only MTHFR C677T homozygous mutation has been considered as thrombotic risk factor in our country [2124]. The frequency of MTHFR C677T homozygous mutation in our study group was similar as compared with our healthy population ( 6.25 vs. $6.30 \%)$. The frequency of MTHFR A1298C heterozygous mutation was $58.1 \%$ and the frequency of homozygous mutation was $8.6 \%$ in healthy Turkish population [21-24]. The frequency of MTHFR A1298C homozygous mutation in our study group was similar as compared with our healthy population (7.7 vs. $8.6 \%$ ).

Furthermore, 8 children $(47.0 \%)$ with NS had three or more risk factors for thrombosis in this study. The patients with three or more risk factors can develop thrombosis easier than those with one or two risk factors [25]. This case series indicates that NS children with additional clinical and/or laboratory risk factors can develop thrombosis easier than others. We suggest that NS children with additional clinical and/or laboratory risk factors should be closely followed up for the development of thrombosis.

In this study, subtype of FSGS, active disease state of NS, central venous catheters, and some inherited and acquired risk factors have been identified in children with NS. Unlike other cohorts, in our case series, FSGS is associated with the highest incidence of thromboembolic complications. FSGS seems to increase thromboembolic risk in children rather than membranous glomerulonephritis or MCNS. There is limited data to support routine prophylactic anticoagulant therapy in children with NS. We could suggest prophylactic anticoagulant therapy for only children with FSGS and three or more risk factors for thrombosis. 
Conflict of interest The authors have declared that no conflict of interest exists.

\section{References}

1. Eddy AA, Symons JM. Nephrotic syndrome in childhood. Lancet. 2003;362:629-39.

2. Kerlin BA, Blatt NB, Fuh B, Zhao S, Lehman A, Blanchong C, et al. Epidemiology and risk factors for thromboembolic complications of childhood nephrotic syndrome: a midwest pediatric nephrology consortium (MWPNC) study. J Pediatr. 2009; 155:105-10.

3. Kerlin BA, Ayoob R, Smoyer WE. Epidemiology and pathophysiology of nephrotic syndrome-associated thromboembolic disease. Clin J Am Soc Nephrol. 2012;7:513-20.

4. Tizki S, Lasry F, Khalifa HH, Itri M. Primary focal segmental glomerular sclerosis in children: epidemiology and prognosis. Nephrol Ther. 2013;9:433-7.

5. Gokce M, Altan I, Unal S, Kuskonmaz B, Aytac S, Cetin M, et al. Recurrent pediatric thrombosis: the effect of underlying and/or coexisting factors. Blood Coagul Fibrinolysis. 2012;23:434-9.

6. Orkin SH, Nathan DG, Ginsburg D, Look AT, Fisher DE, Lux SE. Reference values in infancy and childhood. Nathan and Oski's hematology of infancy and childhood. 7th ed. Philadelphia: Saunders, Elsevier; 2009. p. 1771-96.

7. Gürgey A, Mesci L. The prevalence of factor V Leiden (1691 $\mathrm{G} \rightarrow \mathrm{A}$ ) mutation in Turkey. Turk J Pediatr. 1997;39:313-5.

8. Gürgey A, Hicsönmez G, Parlak H, Balta G, Celiker A. Prothrombin gene 20210 G-A mutation in Turkish patients with thrombosis. Am J Hematol. 1998;59:179-80.

9. Balta G, Gürgey A. Methylenetetrahydrofolate reductase (MTHFR) C677T mutation in Turkish patients with thrombosis. Turk J Pediatr. 1999;41:197-9.

10. Balta G, Altay C, Gurgey A. PAI-1 gene 4G/5G genotype: a risk factor for thrombosis in vessels of internal organs. Am J Hematol. 2002;71:89-93.

11. Bilginer Y, Ozaltin F, Duzova A, Erdogan I, Aki TF, Demircin $\mathrm{M}$, et al. Right atrial thrombosis complicating renal transplantation in a child. Pediatr Transplant. 2008;12:251-5.

12. Topaloğlu R, Saatçi U, Bakkaloğlu A, Beşbaş N, Başsoy Y. Evaluation of the hypercoagulable state by measuring protein $\mathrm{C}$ and antithrombin III levels in nephrotic syndrome and in familial mediterranean fever-related amyloidosis. Turk J Pediatr. 1992;34:15-20.

13. Loscalzo J. Venous thrombosis in the nephrotic syndrome. N Engl J Med. 2013;368:956-8.

14. Rabelink TJ, Zwaginga JJ, Koomans HA, Sixma JJ. Thrombosis and hemostasis in renal disease. Kidney Int. 1994;46:287-96.

15. Abella EM. Hemostatic problems associated with renal disease. Int J Pediatr Hematol Oncol. 1994;1:43-51.

16. Hoyer PF, Gonda S, Barthels M, Krohn HP, Brodehl J. Thromboembolic complications in children with nephrotic syndrome. Acta Pediatr Scand. 1986;75:804-10.

17. Lilova MI, Velkovski IG, Topalov IB. Thromboembolic complications in children with nephrotic syndrome in Bulgaria (1974-1996). Pediatr Nephrol. 2000;15:74-8.

18. Zhang Q, Zeng C, Fu Y, Cheng Z, Zhang J, Liu Z. Biomarkers of endothelial dysfunction in patients with primary focal segmental glomerulosclerosis. Nephrology (Carlton). 2012;17:338-45.

19. Tkaczyk M, Czupryniak A, Owczarek D, Lukamowicz J, Nowicki M. Markers of endothelial dysfunction in children with idiopathic nephrotic syndrome. Am J Nephrol. 2008;28:197-202.

20. Gurgey A, Balta G, Gumruk F, Altay C. Analysis of some clinical and laboratory aspects of adolescent patients with thrombosis. Blood Coagul Fibrinolysis. 2004;15:657-62.

21. Boduroglu K, Alikasifoglu M, Anar B, Tuncbilek E. $677 \rightarrow$ CT mutation on the methylenetetrahydrofolate reductase gene is not a risk factor for neural tube defects in Turkey. Arch Dis Child Fetal Neonatal Ed. 1998;78:F235.

22. Akar N, Akar E, Deda G, Sipahi T, Orsal A. Factor V1691 G-A, prothrombin $20210 \mathrm{G}-\mathrm{A}$, and methylenetetrahydrofolate reductase677 C-T variants in Turkish children with cerebral infarct. J Child Neurol. 1999;14:749-51.

23. Balta G, Gürgey A. Methylenetetrahydrofolate reductase (MTHFR) C677T mutation in Turkish patients with thrombosis. Turk J Pediatr. 1999;41:197-9.

24. Boduroğlu K, Alanay Y, Alikaşifoğlu M, Aktaş D, Tunçbilek E. Analysis of MTHFR 1298A $>$ C in addition to MTHFR 677C $>$ T polymorphism as a risk factor for neural tube defects in the Turkish population. Turk J Pediatr. 2005;47:327-33.

25. Manco-Johnson MJ. How i treat venous thrombosis in children. Blood. 2006;2006(107):21-9. 